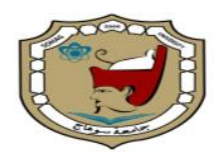

Sohag University

\title{
Can Mosquitos' bites block the hypothesized species jump which leads to the emergence of new viruses? A hypothesis, after reviewing history and geography.
}

\author{
Hassan Mohammed Elnady ${ }^{1}$, Ali Abdelrahman Ghweil ${ }^{2,}$ Tamer Mohamed \\ Mahmoud Abdellah ${ }^{3}$, Ashraf Khodeary ${ }^{4}$ Safaa Khalaf ${ }^{5}$, Mahmoud Saif-Al- \\ Islam $^{5}$
}

1-Department of Neurology and psychological medicine, Faculty of Medicine, Sohag University, Sohag, Egypt.

2-Tropical Medicine and Gastroenterology Department, Faculty of Medicine, South Valley

University, Qena, Egypt.

3-Medical Microbiology and Immunity Department, Faculty of Medicine, Sohag University,

Sohag, Egypt.

4-Clinical Pathology Department, Faculty of Medicine, Sohag University, Sohâg, Egypt.

5-Tropical Medicine and Gastroenterology Department, Faculty of Medicine, Sohag University,

Sohag, Egypt.

\begin{abstract}
Some people have no choice but to get in contact with animals, but this contact is accused of being the main cause of the emergence of new viruses. Novel and creative ways of thinking are needed to reveal the mystery of the reasons that led to the emergence of the viral epidemics and try to treat them.

In our opinion, humankind has been exposed to viral pandemics or at least viral outbreaks in places where, and times when, humans have come close to achieving success in eliminating mosquitoes. And we think that the temporal and local linking between the most important mosquito elimination procedure (which is fighting malaria) and the occurrence of viral pandemics will help in answering many of the questions that have been asked about these pandemics.

For viruses, "Species jumps", a jump between one host species and humans is one of the main steps in the emergence process. Not all mosquito bites are similar; three possibilities can occur to a human or animal after a mosquito bite depending on the mosquito's health status. We hypothesize that one category of mosquito bites stands as a front block against the "Species jumps". This blocking wall will fall if mosquitoes are eliminated which will open the way for new viruses to emerge.

Our hypothesis assumes that mosquitoes are "natural vaccinators", as long as humans can get rid of insect-borne diseases in ways other than vector eradication.
\end{abstract}

Keywords: Mosquitos, species jump, viruses,

\section{INTRODUCTION}

Some people have no choice but to get in contact with animals, but this contact is a- ccused of being the main cause of the emergence of new viruses. Novel and creat- 
ive ways of thinking are needed to reveal the mystery of the reasons that led to the emergence of the viral epidemics and try to treat them.

In our opinion, humankind has been exposed to viral pandemics or at least viral outbreaks in places where, and times when, humans have come close to achieving success in eliminating mosquitoes. And we think that the temporal and local linking between the most important mosquito eliminating procedure (which is fighting malaria) and the occurrence of viral pandemics will help in answering many of the questions that have been asked about these pandemics.

Malaria is a serious disease caused by parasites of the genus Plasmodium that is spread by the bite of an infected female Anopheles mosquito to humans. Epidemiologists were cautiously hopeful that malaria could be prevented if the 'evil' Anopheles were killed or individuals were taken away or shielded from them ${ }^{[1]}$. However, this theory has been challenged (i.e., should the messenger (mosquito) be eradicated when the message (pathogens and parasites) is the enemy of public health? ) [2].

Eradicating mosquitoes (like any other organism) would have serious consequences for ecosystems. Long before man, mosquitoes inhabited this world. The earliest fossils of mosquitoes date from around 200 million years ago. More than 3,500 species of mosquitoes from different parts of the world have now been identified, of which only a few hundred species bite or bother humans. Only female mosquitoes actually bite humans. In various ecosystems, mosquitoes perform essential roles, acting as food for many species, helping to survive filter detritus for plant life, pollinating flowers, and even influencing caribou's herding paths in the tundra. Finally, experts are looking at the mosquito for alternative medicinal therapies ${ }^{[3]}$.

The malaria eradication process is nonselective and affects not only the mosquito strains responsible for the transmission of malaria but even more than that, as it affects a number of biological systems including humans ${ }^{[4]}$.

There is no doubt that some mosquito bites cause several diseases, but it seems that excessive prevention of them will contribute to the emergence of new diseases which develop into catastrophic pandemics over time, and this can be seen through historical analysis.

\section{Spanish flu, 1918.}

By 1914, Europe and the USA had made great strides in malaria which was a major accomplishment given that the anopheline mosquito cycle was only being recognized around 1900, and only recently were the essential vectors and the developmental cycle in the red cell identified ${ }^{[5,6]}$. The four years of the First World War (1914-1918) were a pivotal period in the history of mankind with regard to the elimination of malaria. During the war, innovative methods were produced that improved realistic and tactical techniques. Among them were frequent pyrethrum spraying to control mosquitoes and aggressive mosquito breeding control $^{[5]}$.

A mysterious and lethal epidemic (the Spanish flu) swept across the world in three successive waves (spring 1918, autumn 1918, and winter 1918-19) at the end of this time in 1918. About a third of the world's population was affected by this pandemic and an estimated 50 million people were killed. There was the undoubted consensus of Spanish origin at the time, though army camps in the USA have been blamed more recently. Whatever the precise "Western" origins of the virus, most 
recent authorities accept that the infection of the virus from 1918 to 1919 did not originate in China or the Far East, but instead spread eastward to China ${ }^{[7]}$.

In the absence of air travel at that time, the very large geographical spread of the Spanish flu in such a short period indicated to the authors of a previous report that the epidemic had spread across the globe before this time and that earlier 'seeding' had occurred. The authors have also succeeded in recovering research showing that many related (limited-scale) epidemics existed in different regions of Europe starting in $1916^{[8]}$.

Our explanation for the widespread of the Spanish flu is that it happened in the colonial era and consequently many European countries implemented malaria control measures within their geographical borders as well as within their colonies.

When comparing what happened in India and China during this pandemic, we found that India was severely affected, as nearly 20 million patients died, while China was affected slightly, as the impact of cases was minimal and the number of deaths was small ${ }^{[9]}$. Here it is worth noting that India as a British colony at that time was taking measures to eliminate malaria ${ }^{[10]}$, whilst China did not take similar action ${ }^{[11]}$.

During the 1918 pandemic, the simultaneous outbreaks of influenza in humans and pigs inevitably raised concerns about whether the virus had spread from pigs to humans, or from humans to pigs. So far, this question has not been conclusively answered ${ }^{[12]}$.

We assume that ecosystem violations caused by the over-elimination of mosqueitoes have had immune implications for humans and animals alike.

we think that the Spanish flu pandemic's successive waves were attributable to the continuing battle against malaria during the period of the pandemic, and we think that somehow the environmental balance was restored, which led to the pandemic stopping in late 1919.

\section{Asian influenza, 1957}

Most attention is currently being centered on China and Hong Kong as a possible cause of a new viral pandemic. There have been many explanations for this, including the perfect opportunities for viral transmission via the respiratory route for a large and young human population living under crowded conditions ${ }^{[7]}$.

These reasons were, in our opinion, ineffective before the implementation of anti-malaria measures in this region of the world, and proof of this is the slight impact of the Spanish flu pandemic which occurred in China ${ }^{[9]}$.

We think that the most effective event in China was in 1950 when the anti-malaria campaigns were launched, and slowly, these campaigns started to achieve some successes until the year 1957, when the Asian flu pandemic appeared ${ }^{[11]}$.

This period also witnessed the beginning of the global spread of dichlorodiphenyltrichloroethane (DDT), which started to spread globally at the end of the Second World War. DDT has been used as a pesticide not only to destroy malaria-carrying mosquitoes but also to kill other vectors [13].

Originating in late February 1957 in the Kweichow province of China, the Asian Influenza pandemic spread in less than 4 months to more than 20 countries ${ }^{[14]}$.

\section{The Hong Kong flu (1968 flu pandemic)}

A new influenza pandemic erupted in Southeast Asia in 1968 and gained the sobriquet Hong Kong influenza based on the location of its arrival to Western notice [15]. 
The Hong Kong strain of influenza virus has spread extensively around the world, with many of the countries in which it has been identified not suffering traditional global epidemics, and with many of those in which epidemics have occurred, there was little to no impact on the absence fro$\mathrm{m}$ work and mortality rates. The United States of America was an exception to the general rule, as high illness and death rates were observed after the introduction of the virus on the West Coast ${ }^{[15]}$. When we look at Hong Kong's history of malaria control, we find that it has been a slow process. The tale began in 1930 when a dedicated 'Malaria Bureau' was created. In 1969, the eradication of indigenous cases was successfully carried out [16]. And so, we can discern that the period of time leading up to the Hong Kong pandemic has witnessed successes with regard to the eradication of mosquitoes. What we think of the role of mosquitoes in strengthening human immunity against viral infection makes us think that the countries that have had the greatest success in fighting mosquitoes are the ones that suffered the most when the virus reached them, and at the head of these countries is the United States.

Beginning in the second decade of the twentieth century, and to this day, the United States has tidy methods of controlling mosquitoes, not only at the level of government but also at the public level. Nine out of every ten households in the United States used certain types of pesticides (the vast majority being insecticides) in their house, garden, or yard ${ }^{[6,17,18]}$.

\section{Russian Flu, 1977(red flu)}

Following the beginning of anti-malaria campaigns in the 1950s, China experienced many setbacks and resurgences of malaria that occurred in the 1960s and early 1970 s, but great progress was made after resurgences ${ }^{[19]}$.

Following the effort made after the early seventies, a viral epidemic has emerged (i.e. Russian flu). In November 1977, in the Soviet Union, the Russian (or later, red flu) first came to notice. It was, however, later recorded as having first occurred in May of that year in China. It soon became clear that this rapidly spreading outbreak was almost entirely confined to people under 25 years of age and that the illness was usually mild, albeit marked by typical influenza symptoms ${ }^{[15]}$.

There are many questions asked about the red flu epidemic and have not been answered so far ${ }^{[15]}$.

Regarding the age group the virus selected, we think the reason is that it was this age group that was born and lived in conjunction with the widespread use of DDT after the early 1950s. This age group did not take its share of insect bites as the previous age groups.

\section{The Ebola outbreaks}

The first recorded Ebola outbreak occurred in northern Zaire in 1976 (now the Democratic Republic of Congo). It occurred near the Ebola River after which the virus was named. 318 cases with a case fatality of 88 percent were found in total. A similar outbreak occurred in the south of Sudan two months prior to the 1976 outbreak in Zaire. These two outbreaks were initially thought to be related owing to the proximity of the subsequent outbreak in Zaire. However, the causative agents were eventually found to be two distinct strains of the Ebola virus, later named the Zaire and Sudan strains, respectively ${ }^{[20]}$. With regard to the country called Zaire at that time, we will find that the first measures to combat malaria began in the form of vector control measures ${ }^{[21]}$. In the light of Sudan, it is noteworthy 
that the period beginning in the 1970s and later saw remarkable activity in malaria control measures ${ }^{[22]}$.

Since Ebola was first reported in 1976, there have been 29 outbreaks or case reports of Ebola virus disease (EVD) documented. Ebola has been known to cause outbreaks in central and eastern Africa for a long period of time, as no sporadic human cases or outbreaks have been recorded in West Africa before. But West Africa began to be affected in $2013^{[20]}$.

In West Africa, the 2013-2016 epidemic was unprecedented, being larger than all past outbreaks combined. In its geographical distribution and multi-country spread, it was also peculiar ${ }^{[20]}$.

In the West African epidemic, the majority of cases were concentrated in three countries with heavy transmission: Sierra Leone, Liberia, and Guinea ${ }^{[20]}$.

If the African malaria eradication measures were traced back to the 1970 s, a temporal and spatial association with these numerous and unprecedented Ebola outbreaks will be found.

\section{The Middle East respiratory syndrome (MERS) 2012}

Lover and colleagues reported that it was considered useful to draw attention to the common reality that if malaria eradication is theoretically feasible, it is and will always remain a rather serious challenge, and that policymakers should not underestimate their challenges or expect that any of the problems will be resolved automatically as the year's pass ${ }^{[23]}$.

Thus, Countries have often brought about improvements in malaria control programs, and it is difficult to judge the ability of these improvements to eliminate mosquitoes for several factors. In addition, the time span for any success may extend for many years. Therefore, the period of time in which, there is a stable decline in malaria cases, can be considered to be the period with the best successes.

Regarding Saudi Arabia, in 2004, a revised action plan was developed, to eliminate malaria. The period between 2008 and 2014 witnessed a steady decline in cases in a certain locality in Saudi Arabia ${ }^{[24]}$. In conjunction with this period, a viral epidemic occurred in Saudi Arabia (i.e. Middle East Respiratory Syndrome) ${ }^{\text {[25] }}$.

The Middle East respiratory syndrome (MERS) is a highly lethal respiratory disease caused by betacoronavirus (MERSCoV), a single-stranded, positive-sense RNA virus. In Jeddah, Saudi Arabia, the virus was first isolated from a patient who died from a serious respiratory disease in June 2012. While most cases of MERS have occurred in Saudi Arabia and the United Arab Emirates, cases of people traveling from the Middle East or their contacts have been recorded in Europe, the USA, and Asia ${ }^{[25]}$.

It has been verified by many studies from several Middle Eastern countries (with the exception of Yemen, a country that suffers a lot from malaria ${ }^{[26]}$ ) that camels are the source of the infection [27]. It should be remembered that, during this period of time, the camel, whose name has been associated with health and cure in the minds of the inhabitants of the Arabian Peninsula since ancient times ${ }^{[28]}$, became a source of serious illness.

\section{COVID-19, 2019}

In China, the National Malaria Elimination Action Plan (NMEAP) (2010-2020) was released with the goal of eliminating indigenous malaria before the end of 2015 in non-border regions and eliminating the disease nationally before the end of 2020. Among the successful methods that led to a decrease in the incidence of malaria cases were indoor residual spr- 
aying and the distribution of insecticidal nets ${ }^{[29]}$.

In December 2019, an unexplained outbreak of pneumonia of unknown etiology occurred in Wuhan City, Hubei Province, China. The causative agent was described as a novel coronavirus and was subsequently named COVID-19 by the World Health Organization (WHO). The incidence of COVID-19 continues to increase, despite stringent global containment and quarantine efforts ${ }^{[30]}$.

\section{Hypothesis and argument.}

More than two-thirds of human virus species are zoonotic, and a subset (less than 20\%) of zoonotic viruses are also believed to infect birds; relatively few vertebrates other than mammals or birds have been recorded. As far as we are aware, the remaining viruses infect humans only naturally (these are often referred to as 'specialist' human pathogens. There are 219 species of viruses known to be able to kill human beings. There are already three or four new species discovered per year. Extrapolation of the detection curve indicates that a large reservoir of undiscovered human virus species still exists ${ }^{[31]}$.

A previous study attempted to determine the minimum number of viruses expected to emerge and infect humans, with the results suggesting that there are at least 320,000 different viruses [32].

How many vaccinations will any person need to be protected from future viral diseases? And will it be reasonable? There is inevitably a natural way of preventing the emergence of these new viruses.

Woolhouse and colleagues ${ }^{[33]}$ reported that, For viruses, "Species jumps", a jump between one host species and humans is one of the main steps in the emergence process (for more details see ${ }^{[33]}$ ).
We hypothesize that mosquitoes" bites are one of the natural processes that can prevent species jumps and therefore have the ability to prevent the emergence of new viruses.

This is attributed to the widely researched reinforcing role of human immunity played by mosquito bites ${ }^{[34-38]}$.

Of the over 545 suspected arbovirus species more than 150 are documented to cause disease in humans, and the majority are zoonotic ${ }^{[39]}$. Previous studies have discussed the possibility of mosquitoes playing a role in transmitting certain viral diseases not belonging to arboviruses. These studies could not deny that small doses of these viruses, killed viruses or viral particles could be transmitted by mosquitoes [40].

Therefore, we can say that mosquitoes can transmit a group of viruses belonging to the arboviruses known to cause diseases in humans, and we will define them in this article as "Pathogenic Viruses".

Moreover, mosquitoes can also transmit hundreds of arboviruses not known to cause diseases in humans, and an unknown number of viruses (but it seems to be a huge number because it definitely includes a large collection of non-emergent viruses) that do not belong to arboviruses, and we will define them in this article as "non Pathogenic Viruses" (even if they causing a human disease via other methods of transmission).

So, not all mosquito bites are similar, there are three possibilities that can occur to a human or animal after a mosquito bite depending on the mosquito's health status:

a) The first possibility is when the mosquito is healthy, whereupon only the mosquito's saliva enters human or animal blood. 
b) The second possibility is when the mosquito is infected with "Pathogenic Viruses," which causes arthropod-borne viral diseases to occur.

c) The third possibility is when the mosqueito is infected with "non Pathogenic Viruses"; inevitably this will lead to the formation of immune reactions against these viruses, including the large collection of non-emergent viruses. And if we add the so-called cross-immunity, we can say that this

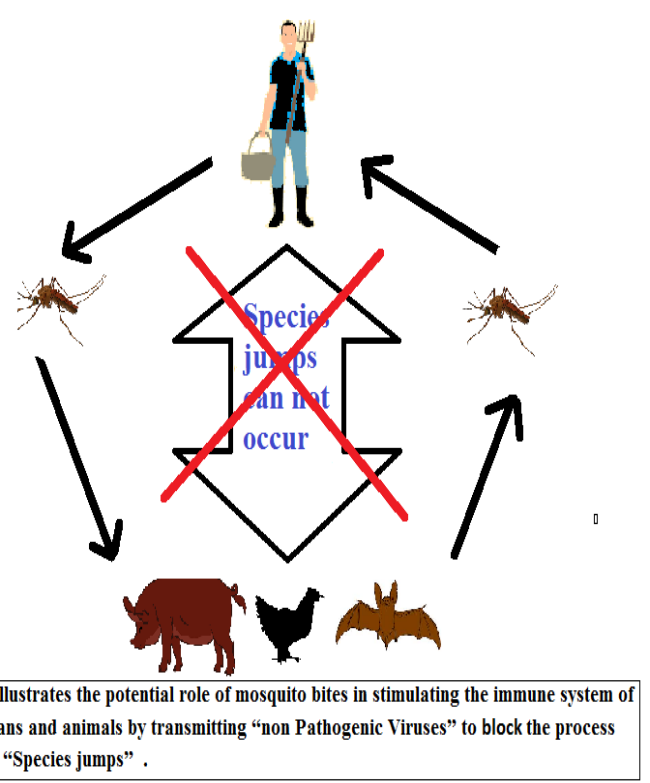

\section{References}

1. Gachelin, G. and A. Opinel, Malaria epidemics in Europe after the First World War: the early stages of an international approach to the control of the disease. Hist Cienc Saude Manguinhos, 2011. 18(2): p. 431-69.

2. Harbach, R.E. and N.J. Besansky, Mosquitoes. Curr Biol, 2014. 24(1): p. R14-r15.

3. Hadley, D. What Good Are Mosquitoes? 2019 Updated July 03, 2019; Available from thoughtco.com/what-good-aremosquitoes-1968303.

4. Bouwman, H., H. van den Berg, and H. Kylin, DDT and malaria prevention: addressing the paradox. Environmental type of mosquito bites stands as a front block against the "Species jumps" [Figure 1]. This blocking wall will fall if mosquitoes are eliminated, which will open the way for new viruses to emerge [Figure 2].

Our hypothesis assumes that mosquitoes are "natural vaccinators", as long as humans are able to get rid of insect-borne diseases in ways other than vector eradication.

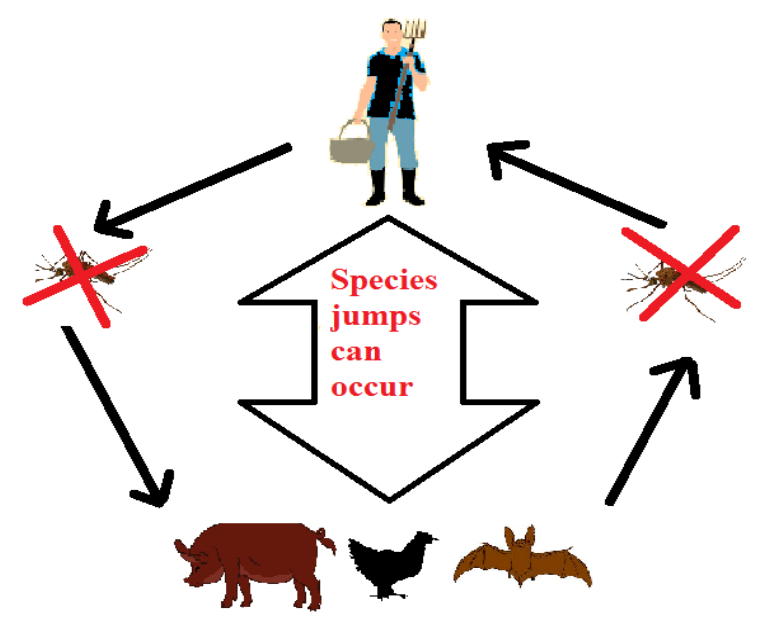

Figure No. 2 shows that in the absence of the potential role of mosquito bites in stimulating the immune system (due to the excessive elimination of mosquitoes) of both humans and animals by transmitting "non Pathogenic Viruses" the process known as "Species jumps" is not blocked resulting in the emergence of new viruses.

health perspectives, 2011. 119(6): p. 744747.

5. Brabin, B.J., Malaria's contribution to World War One - the unexpected adversary. Malar J, 2014. 13: p. 497.

6. Williams, L.L., Jr., Malaria eradication in the United States. Am J Public Health Nations Health, 1963. 53: p. 17-21.

7. Oxford, J.S., et al., A hypothesis: the conjunction of soldiers, gas, pigs, ducks, geese, and horses in northern France during the Great War provided the conditions for the emergence of the "Spanish" influenza pandemic of 19181919. Vaccine, 2005. 23(7): p. 940-5. 
8. Oxford, J.S., The so-called Great Spanish Influenza Pandemic of 1918 may have originated in France in 1916. Philosophical transactions of the Royal Society of London. Series B, Biological Sciences, 2001. 356(1416): p. 1857-1859.

9. Cheng, K.F. and P.C. Leung, What happened in China during the 1918 influenza pandemic? Int $\mathrm{J}$ Infect Dis, 2007. 11(4): p. 360-4.

10.Hehir, P., Prevention of Malaria in the Troops of Our Indian Empire. Ind Med Gaz, 1914. 49(8): p. 305-309.

11.Zhou, Z.J., The malaria situation in the People's Republic of China. Bull World Health Organ, 1981. 59(6): p. 931-6.

12.Nelson, M.I. and M. Worobey, Origins of the 1918 Pandemic: Revisiting the Swine "Mixing Vessel" Hypothesis. Am J Epidemiol, 2018. 187(12): p. 2498-2502.

13.Yeo, I.S., U.S. military administration's malaria control activities (1945-1948). Uisahak, 2015. 24(1): p. 35-65.

14.Cobos, A.J., et al., Mortality and transmissibility patterns of the 1957 influenza pandemic in Maricopa County, Arizona. BMC infectious diseases, 2016. 16(1): p. 405-405.

15. Kilbourne, E.D., Influenza pandemics of the 20th century. Emerg Infect Dis, 2006. 12(1): p. 9-14.

16.Sinha, R., Malaria in Hong Kong: Impact, eradication, and legacy. International Journal of Infectious Diseases, 2016. 45: p. 382-383.

17.Stoops, C.A., et al., A Review of Studies Evaluating Insecticide Barrier Treatments for Mosquito Control From 1944 to 2018. Environ Health Insights, 2019. 13: p. 1178630219859004.

18.Savage, E.P., et al., Household pesticide usage in the United States. Arch Environ Health, 1981. 36(6): p. 304-9.

19.Huang, S. and author_in_Japanese, THE PRESENT STATUS OF MALARIA CONTROL IN THE PEOPLE'S REPUBLIC OF CHINA. Japanese Journal of Tropical Medicine and Hygiene, 1980. 8(3-4): p. 159-172.
20.Coltart, C.E., et al., The Ebola outbreak, 2013-2016: old lessons for new epidemics. Philos Trans R Soc Lond B Biol Sci, 2017. 372(1721).

21.Lechthaler, F., et al., Trends in reported malaria cases and the effects of malaria control in the Democratic Republic of the Congo. PLOS ONE, 2019. 14(7): p. e0219853.

22.Noor, A.M., et al., Malaria risk mapping for control in the Republic of Sudan. The American journal of tropical medicine and hygiene, 2012. 87(6): p. 1012-1021.

23.Lover, A.A., et al., Malaria Elimination: Time to Target All Species. Am J Trop Med Hyg, 2018. 99(1): p. 17-23.

24.Alshahrani, A.M., et al., The changing malaria landscape in Aseer region, Kingdom of Saudi Arabia: 2000-2015. Malar J, 2016. 15(1): p. 538.

25.Zumla, A., D.S. Hui, and S. Perlman, Middle East respiratory syndrome. Lancet, 2015. 386(9997): p. 995-1007.

26.Anam, L.S., et al., Evaluation of Two Malaria Surveillance Systems in Yemen Using Updated CDC Guidelines: Lessons Learned and Future Perspectives. Inquiry, 2019. 56: p. 46958019880736.

27.Al-Osail, A.M. and M.J. Al-Wazzah, The history and epidemiology of Middle East respiratory syndrome coronavirus. Multidiscip Respir Med, 2017. 12: p. 20.

28. Abdel Gader, A.G.M., and A.A. Alhaider, The unique medicinal properties of camel products: A review of the scientific evidence. Journal of Taibah University Medical Sciences, 2016. 11(2): p. 98-103.

29.Lai, S., et al., Changing epidemiology and challenges of malaria in China towards elimination. Malar J, 2019. 18(1): p. 107.

30.Sohrabi, C., et al., World Health Organization declares global emergency: A review of the 2019 novel coronavirus (COVID-19). Int J Surg, 2020. 76: p. 7176.

31.Woolhouse, M., et al., Human viruses: discovery and emergence. Philos Trans R Soc Lond B Biol Sci, 2012. 367(1604): p. 2864-71. 
32.Anthony, S.J., et al., A Strategy To Estimate Unknown Viral Diversity in Mammals. mBio, 2013. 4(5): p. e0059813.

33. Woolhouse, M.E.J., D.T. Haydon, and R. Antia, Emerging pathogens: the epidemiology and evolution of species jumps. Trends in ecology \& evolution, 2005. 20(5): p. 238-244.

34.Vogt, M.B., et al., Mosquito saliva alone has profound effects on the human immune system. PLoS Negl Trop Dis, 2018. 12(5): p. e0006439.

35.Manning, J.E., et al., Mosquito Saliva: The Hope for a Universal Arbovirus Vaccine? J Infect Dis, 2018. 218(1): p. 715.

36.Samuel, G.H., Z.N. Adelman, and K.M. Myles, Antiviral Immunity and VirusMediated Antagonism in Disease Vector
Mosquitoes. Trends Microbiol, 2018. 26(5): p. 447-461.

37.Blair, C.D., Mosquito RNAi is the major innate immune pathway controlling arbovirus infection and transmission. Future Microbiol, 2011. 6(3): p. 265-77.

38.Billingsley, P.F., et al., Immune interactions between mosquitoes and their hosts. Parasite Immunol, 2006. 28(4): p. 143-53.

39. Cleton, N., et al., Come fly with me: a review of clinically important arboviruses for global travelers. J Clin Virol, 2012. 55(3): p. 191-203.

40.Chang, T.T., et al., Existence of hepatitis $\mathrm{C}$ virus in Culex quinquefasciatus after ingestion of infected blood: an experimental approach to evaluating transmission by mosquitoes. J Clin Microbiol, 2001. 39(9): p. 3353-5. 